\title{
Cardiorenal biomarkers in acute heart failure
}

\author{
Rajiv Choudhary ${ }^{1}$, Dipika Gopal ${ }^{2}$, Ben A. Kipper ${ }^{1}$, Alejandro De La Parra Landa ${ }^{1}$, Hermineh Aramin ${ }^{1}$ \\ Elizabeth Lee $^{1}$, Saloni Shah ${ }^{1}$, Alan S. Maisel ${ }^{1,2}$ \\ ${ }^{1}$ VA Medical Center, 3350 La Jolla Village Drive, San Diego, CA 92103, USA \\ ${ }^{2}$ Department of Medicine, University of California at San Diego, San Diego, CA 92103, USA
}

\begin{abstract}
Managing patients with heart failure (HF) is a challenging task within itself, but the presence of associated worsening renal function can greatly increase mortality and morbidity. Early diagnosis and treatment is the key to prevent re-hospitalizations and reduce healthcare costs. Biomarkers have long been established as highly sensitive and specific tools in diagnosing and prognosticating patients with HF. Reflecting distinct pathophysiological events and ongoing cellular insult, biomarkers have been proven superior to conventional laboratory tests. Availability of better assays and rapid analysis has allowed the use of biomarkers as point-of-care tests in the emergency department and at the patient's bed-side. Acute HF patients often go on to develop worsening renal function, termed as acute cardiorenal syndrome. The growing breadth of studies has shown the implications of combining multiple biomarkers to better chart outcomes and produce desirable results in such patients.
\end{abstract}

J Geriatr Cardiol 2012; 9: 292-304. doi: 10.3724/SP.J.1263.2012.02291

Keywords: Heart failure; Cardiorenal biomarkers; Acute kidney injury

\section{Introduction}

Acute heart failure (HF) is the leading cause of hospitalizations in the age group 65 years and over and represents a significant economic burden. ${ }^{[1-3]}$ With a prevalence of $2 \%$, HF admissions have surpassed the one million mark in the U.S. Healthcare costs associated with managing HF have crossed $\$ 39$ billion dollars and require an integrated approach to control the growing expenses. ${ }^{[2]}$ One of the problematic areas in managing patients with HF is the involvement of multiple organs and presence of multiple co-morbidities. The kidney plays a vital role in maintaining hemodynamic balance, which is often disrupted in HF patients. Communication between the heart and kidneys is affected in acute $\mathrm{HF}$, resulting in decreased organ perfusion and ultimately organ failure and death. Acute HF is characterized by diminished left ventricular systolic function and poor cardiac output triggering compensatory mechanisms such as the reninangiotensin-aldosterone system, the sympathetic system, and other local mediators, which interact to maintain the fluid

Correspondence to: Rajiv Choudhary, MD, MPH, VA Medical Center, 3350 La Jolla Village Drive, San Diego, CA 92103, USA.

E-mail: dr.rajive@gmail.com

Telephone: +1-858-657-5318

Fax: $+1-858-657-8032$

Received: February 29, 2012

Revised: May 28, 2012

Accepted: June 5, 2012

Published online: August 28, 2012 volume. ${ }^{[2-4]}$ The imbalance between these compensatory mechanisms, unable to maintain adequate cardiac output results in volume overload. Furthermore, decreased renal perfusion and in addition to nephrotoxic agents and over-diuresis, eventually leads to acute kidney injury (AKI) in such patients. Novel techniques such as the use of biomarkers and bio-impedance analysis has received considerable attention in the diagnosis and prognosis of patients with $\mathrm{HF}$ and worsening renal function (Figure 1). Biomarkers are secreted in response to increased "stress" and are widely used to monitor progression and severity of disease. Their ability to reflect distinct pathophysiological changes that occur in acute HF and AKI make them superior to conventional markers. This review will provide an update on current and emerging cardiac and renal biomarkers with diagnostic and prognostic implications in HF patients. In discussing markers for cardiac dysfunction in patients with cardiorenal syndrome, natriuretic peptides (NPs) and cardiac troponins will be reviewed. NPs are considered benchmark markers and have set the bar for markers in HF. Markers such as troponin have high prognostic value especially in serial monitoring of their levels to indicate ongoing myocardial injury/ insult, thus being an effective marker for risk stratification and cardiac dysfunction. In discussing markers for renal dysfunction, robust marker such as neutrophil gelatinase associated lipocalin (NGAL) and emerging markers such as Cystatin $\mathrm{C}$ will be reviewed. Finally, prognostic markers such as Procalcitonin

http://www.jgc301.com; jgc@jgc301.com | Journal of Geriatric Cardiology 


\begin{abstract}
Markers of cardiac dysfunction
\end{abstract}

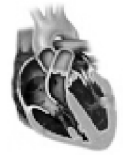

BNP: secreted from cardiac ventricles in response to increased wall stress

Troponins: secreted due to cardiac mayocyte injury/necrosis

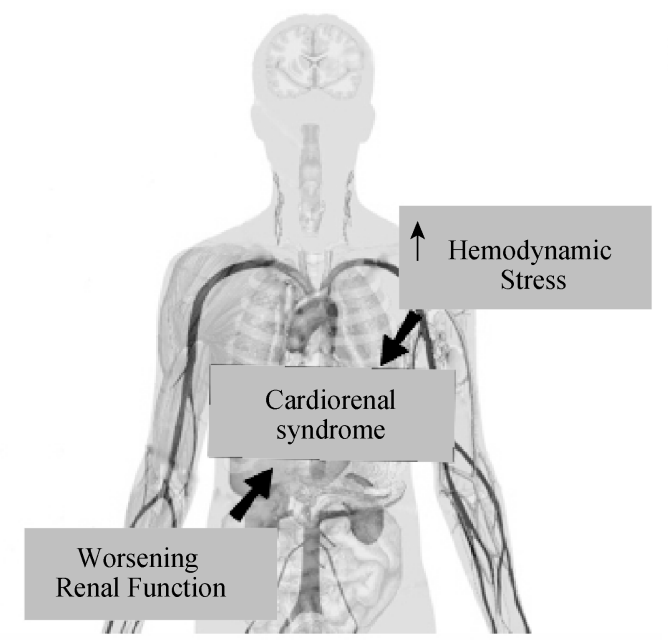

Markers of renal dysfunction

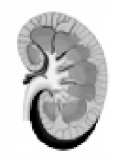

NGAL: Secreted from proxinal tubule cells in response to ischemic injury

Cystatin C: Cysteine proteinase inhibitor, a measure of glomerular filtration rate

Prognostic markers of cardiac dysfunction

Procalcitonin: Marker for concomitant infection seen in patients with heart failure

Trimodal pattern of release from:

-Neuroendorine cells

-Parenchymal tissue (adipose cells)

-Peripheral blood mononuclear cells

In response to:

Endotoxin release \& inflammatory cytokines

\author{
Adrenomedullin: Expressed in endothelial \\ tissues and strong prognostic marker for \\ cardiovascular disease \\ Copeptin: secreted from \\ posterior pituitary in \\ response to decreased blood \\ volume
}

Figure 1. Schematic representation of different sites of biomarker release and expression in the human body. BNP: B-type natriuretic peptide; NGAL: Neutrophil gelatinase associated lipocalin.

(marker for concomitant infection), Adrenomedullin (ADM) and Copeptin (Anti-diuretic hormone) will also be reviewed.

\section{Renal dysfunction in acute HF}

According to acute HF registry databases, renal dysfunction is the most frequent comorbidity in acute HF patients associated with high in-patient mortality. ${ }^{[5,6]}$ In a meta-analysis by Smith et al., ${ }^{[7]}$ unadjusted mortality rate at one year followup was $51 \%$ in those with moderate to severe renal impairment compared to $26 \%$ in those without any renal impairment. Moreover, after adjusting for socio-demographic and clinical covariates, those with any renal impairment were still at significant higher risk of mortality $(\mathrm{HR}=1.56, P<$ $0.001)$. The meta-analysis also demonstrated that those with high degree of HF severity in accordance with NYHA functional class, had a markedly elevated mortality rate if any renal dysfunction was present. Patients with acute HF can be classified as de novo HF and acute decompensated HF (ADHF) depending on onset and duration of their symptoms. ${ }^{[8,9]}$ Patients with de novo HF have no previous history or symptoms and signs of HF and present with their first manifestation of HF, whereas those with ADHF have pre-existing $\mathrm{HF}$ and present due to rapid worsening of their symptoms, usually in a decompensated state with fluid overload. Majority of patients with acute HF have coronary artery disease, hypertension and mitral regurgitation as their three main underlying conditions. ${ }^{[10]}$ Although left ventricular systolic dysfunction is associated with acute HF patients, studies have shown that most of the patients with acute HF may have preserved systolic function with or without diastolic dysfunction. Poor cardiac output as seen in most patients with acute $\mathrm{HF}$ is the main driving factor causing decreased renal perfusion and worsening renal function characterized as cardiorenal syndrome. ${ }^{[10,11]}$ Ronco et al., ${ }^{[1]]}$ proposed the definition for cardiorenal syndrome as, "disorders of the heart and kidneys whereby acute or chronic dysfunction in one organ may induce acute or chronic dysfunction of the other." Furthermore, cardiorenal syndrome is classified into 5 types depending on which primary organ (heart or kidney) is affected first.

\section{Pathophysiology of renal dysfunction in acute HF}

The inter-relation that exists between the heart and kidney is affected in patients with acute HF. ${ }^{[5,6]}$ Low cardiac output results in renal arteriolar vasoconstriction which 
increases central venous pressure and further reduces renal blood flow. ${ }^{[8]}$ Renal hypoperfusion triggers the sympathetic system and activates the renin-angiotensin-aldosterone system to maintain plasma volume by retaining sodium. Adenosine release is triggered by the rising sodium levels which then bind A1-receptors in the proximal tubule leading to afferent glomerular arteriole constriction resulting in glomerular filtration rate (GFR). ${ }^{[12]}$ In patients with long standing HF, rapid worsening of their symptoms leads to rapid worsening of their renal function. For instance, in the acute setting, patients with ADHF present with fluid overload wherein the compensatory mechanism activated result in further deterioration of cardiac and renal function. ${ }^{[12]}$ Biomarkers which are released in response to hemodynamic stress in such cases are useful tools to monitor disease progression and severity. Studies have shown that monitoring biomarkers of cardiac dysfunction in conjunction with markers of renal dysfunction may prove most beneficial in managing such patients.

\section{Diagnostic and prognostic markers of cardiac dysfunction}

\subsection{B-type natriuretic peptide (BNP)}

Acute HF is a debilitating disease with mortality exceeding most cancers and represents one of the most expensive disorders in the medical field. ${ }^{[13]}$ The majority of acute HF patients will eventually require attention in the emergency department or hospital. As a quantitative marker of $\mathrm{HF}$, the use of BNP levels in serial measurements could be useful not only for diagnosis, but also for risk stratification and may therefore assist in triage decisions. BNP is part of a structurally similar family of peptide hormones which also includes: atrial natriuretic peptide (ANP), C-type natriuretic peptide (CNP), and Urodilatin. ${ }^{[14]}$ BNP is a neurohormone consisting of 32 amino-acids synthesized in the cardiac ventricles and is released in response to ventricular dilatation and pressure overload. ${ }^{[15]}$ After synthesis, the precursor peptide is cleaved first to pro-BNP, then to the biologically active BNP and the inactive 76 amino-acid N-terminal fragment (NT-proBNP). The release of BNP results in improved myocardial relaxation and serves an important regulatory role in response to acute increases in ventricular volume by opposing the vasoconstriction, sodium retention and antidiuretic effects of the activated reninangiotensin-aldosterone system. ${ }^{[16]}$ Different pathological states increase BNP plasma concentrations, particularly when there is increased dilatation of the cardiac wall chamber, an expanded fluid volume (HF, renal failure, primary hyperaldosteronism), or reduced clearance of peptides (renal failure). ${ }^{[14]}$

Initial emergency department BNP levels can identify the risk of death or readmission within 30 days. ${ }^{[16]}$ In the REDHOT study, Maisel et al. ${ }^{[17]}$ analyzed a cohort of 464 patients presenting to the emergency department with symptoms suggestive of congestive $\mathrm{HF}$ (CHF). Of these patients, $68.3 \%$ were going to be admitted, but after the initial assessment was performed the rate went up to $90.3 \%$ of the population. The 90 days combined event rate in the group of patients admitted with BNP $<200 \mathrm{pg} / \mathrm{mL}$ was $9 \%$ compared to $29 \%$ in those admitted with BNP $>200 \mathrm{pg} / \mathrm{mL}$. This demonstrates that the overall prognosis of patients with BNP levels $<$ $200 \mathrm{pg} / \mathrm{mL}$ is excellent, despite their classification as NYHA functional class III or IV.

BNP can also be helpful for predicting in-patient mortality, as seen in the ADHERE study. ${ }^{[15]} 48,629$ (63\%) of 77,467 patients had BNP assessments within $24 \mathrm{~h}$ of presentation. Overall, in-hospital mortality was $3.6 \%$, but this mortality risk varied more than 3 to 4 fold on the basis of the patient's initial BNP levels, suggesting that the risk of in-hospital mortality can be reliably estimated using BNP obtained at hospital admission. In another study, patients with acute HF who were discharged based on absolute percentage reduction of BNP from admission by $>46 \%$ at discharge in conjunction with total $\mathrm{BNP}<300 \mathrm{pg} / \mathrm{mL}$ carried lower risk [Odds ratio (OR): $4.77,95 \%$ confidence interval $(95 \% \mathrm{CI}): 1.76-12.83$, $P<0.002$ ] compared to those with $<46 \%$ reduction at discharge and BNP $>300 \mathrm{pg} / \mathrm{mL}$ (OR: 9.61, 95\% CI: 4.5120.46, $P<0.001) \cdot{ }^{[18]}$

Thus BNP is most certainly an integral tool useful in treating and managing patients with acute HF indicative of ongoing disease and allowing to better preserve renal function by timely discharge of patients especially in the emergency department.

\subsection{NT-proBNP}

As mentioned before, the dynamic link between heart and kidney can be affected in patients with acute HF resulting in worsening renal function and deteriorating patient survival. With activation of various compensatory pathways in response to low blood pressure and poor cardiac output, charting NPs levels in conjunction with measuring degree of renal dysfunction can certainly improve outcomes of patients with cardiorenal syndrome. Like BNP, NT-proBNP release from ventricular myocytes is due to increase ventricular pressure and volume overload but with a longer half-life (120 min). Using NTproBNP levels in the diagnosis and risk stratification of patients has proved to be superior to its counterpart. ${ }^{[19]}$ As factors such as renal dysfunction, age, pulmonary disease influence NPs levels, in patients with worsening renal function in the acute setting, elevated NP levels reflect ongoing disease severity and not mere accumulation. ${ }^{[20]}$ As demonstrated in a follow-up to the ICON study by Kimmenade et al. ${ }^{[20]}$ in

\footnotetext{
Journal of Geriatric Cardiology | jgc@jgc301.com; http://www.jgc301.com
} 
720 patients with acute HF, glomerular filtration rate (GFR), and NT-proBNP were the best predictors of short-term 60-day mortality (OR: 3.46, 95\% CI: 2.13-5.63). Moreover, a rise in both NT-proBNP and GFR or serum creatinine levels $(>0.3 \mathrm{mg} / \mathrm{dL})$ were predictive of worse outcomes. The study stated that elevated NT-proBNP levels on admission combined with poor GFR (GFR $<60 \mathrm{~mL} / \mathrm{min}$ per $1.73 \mathrm{~m}^{2}$ ) were more accurate in indicating worse prognosis compared to serial serum creatinine levels. Despite of several limitations, the study demonstrated the prognostic utility of using NT-proBNP in addition to GFR and serum creatinine to better risk stratify patients with cardiorenal syndrome.

In another study, Kimmenade et al. ${ }^{[21]}$ demonstrated that although NT-proBNP levels was unable to discriminate acute HF etiology (ischemic vs. non-ischemic), it proved useful in differentiating the type of HF. NT-proBNP levels were higher in those with systolic $\mathrm{HF}(6,196 \mathrm{pg} / \mathrm{mL})$ compared to those with non-systolic HF (3,134 pg/mL). Also highlighting the importance of inflammatory markers such as apelin and galectin-3 in acute HF patients, receiver operating characteristic (ROC) analysis in predicting short-term mortality demonstrated a superior area under the curve (AUC) for galectin-3 (0.74) compared to NT-proBNP (0.67) and apelin (0.54) in those with acute HF. On adjusted multivariable analysis galectin-3 (OR: 14.3, 95\% CI: 5.6-45.1, $P<0.001$ ) outperformed NT-proBNP and apelin in predicting short-term mortality. The study claimed that NT-proBNP is superior to galectin-3 and apelin in diagnosing acute HF whereas galectin-3 is superior to others in predicting short-term mortality.

Interpreting natriuretic peptide (NP) levels accurately is critical in early diagnosis and management of patients with acute HF. In a follow-up to the PRIDE study, Januzzi et $a l^{[22]}$ demonstrated that in 1,256 patients presenting to the emergency department with dyspnea using appropriate agerelated cut-points in diagnosing acute $\mathrm{HF}$ improved diagnostic performance of NT-proBNP on ROC analysis. In patients < 50 years, ROC analysis achieved an AUC of 0.99 in diagnosing acute $\mathrm{HF}$ followed by 0.93 and 0.86 for ages $50-75$ years old and $>75$ years old, respectively. Using a cut-point of 300 $\mathrm{pg} / \mathrm{mL}$ to exclude diagnosis of acute $\mathrm{HF}$ achieved $99 \%$ sensitivity, $60 \%$ specificity and $98 \%$ negative predictive value. Furthermore, those with elevated NT-proBNP levels had higher risk of short-term mortality (OR: 5.2, 95\% CI: $2.2-8.1 ; P<0.001)$.

The value of using NPs as an indicator of cardiac dysfunction on patients with worsening renal function is obvious. Past research in NPs had allowed a better understanding of underlying pathophysiological changes that occur in patients with acute HF. However, care should be taken while interpreting NP levels since patients with acute HF often present with grey- zone values, making it necessary to appropriately follow-up such patients with serial measures. Using NPs individually or in combination with other parameters such as GFR, serum creatinine, may prolong survival in patients with caridorenal syndrome.

\subsection{Troponins}

Cardiac troponin is released into circulation as a result of cardiac injury and includes troponin I, troponin $\mathrm{T}$ and troponin $\mathrm{C}$ which regulate actin-myosin interaction and regulate calcium mediated muscle contraction. ${ }^{[23]}$ Researchers have reported strong correlations between cardiac troponins and renal function. ${ }^{[24]}$ In the acute setting, wherein majority of acute HF patients present, troponin levels can be a useful surrogate of indicating ongoing inflammation or myocyte injury and worsening renal function; thus charting troponin levels may help identify patients with acute HF at a high risk. Additionally, serum troponin concentration remains high in the blood much longer than creatine kinase-MB, creatine kinase, and myoglobin due to its significantly longer half-life, providing a longer diagnostic period. In-patient mortality in patients with acute HF is often elevated in the presence of poor renal function. Serial troponin measures in patients hospitalized with HF may enable better monitoring of such patients. With ischemic heart disease as a predominant cause triggering acute $\mathrm{HF}$, using troponin levels to measure ongoing myocardial damage can be beneficial in better managing patients with acute HF and renal dysfunction. ${ }^{[25]}$ With the advent of high sensitivity assays for cardiac troponin, a more accurate and rapid detection of an ischemic event is possible.

Kitagawa et al. ${ }^{[26]}$ demonstrated that in patients with chronic kidney disease and diastolic failure, high-sensitivity cardiac troponin $\mathrm{T}$ (hs-cTnT) along with BNP were powerful predictors of detecting left ventricular end-diastolic dysfunction. area under the receiver operating characteristic curve (ROCAUC $)$ is $0.88(P=0.010)$ for hs-cTnT and ROC-AUC is 0.741 ( $P=0.057)$ for BNP. Hs-cTnT significantly correlated with parameters of peak flow velocity across mitral valve.

Cardiac troponin levels has also helped identify patients with $\mathrm{HF}$ and increased risk of in-patient mortality. Nishio et al. ${ }^{[27]}$ studied 145 inpatients (mean ejection fraction $=31.6 \% \pm 0.9 \%$ ) with congestive HF, who were split into groups with high $(\geq 0.1 \mathrm{ng} / \mathrm{mL})$ and low $(<0.01 \mathrm{ng} / \mathrm{mL})$ serum cardiac troponin $\mathrm{T}$ level. Patients with higher cardiac troponin levels also had significantly high serum levels of BNP $(P<0.0001)$. Of the CHF patients in the study, $32 \%$ had high troponin $\mathrm{T}$ levels and $68 \%$ had low troponin $\mathrm{T}$ levels. Of these, $9 \%$ with low troponin $\mathrm{T}$ levels and $41 \%$ with high troponin $\mathrm{T}$ levels died or were re-hospitalized due to $\mathrm{CHF}$. The analyses indicated that high cardiac troponin levels and high BNP levels along 
with other clinical variables were strong indicators of death and re-hospitalization for CHF patients.

De Filippi et al. ${ }^{[28]}$ noted that when troponin T levels are greater than $12.95 \mathrm{pg} / \mathrm{mL}$, the hazard ratio is 2.48 for $\mathrm{CHF}$. The potential of cardiovascular death is highly increased for those with troponin T levels greater than or equal to $12.95 \mathrm{pg} / \mathrm{mL}$ had a hazard ratio of 1.35 to 2.91 .

Although studies have successfully demonstrated the diagnostic and prognostic utility of using both cardiac troponin I \& T levels in patients with acute HF, further research exploring serial troponin measures in patients with renal dysfunction may prove beneficial. The use of hs-cTnT can certainly improve overall survival of acute HF patients by reducing in-hospital stay and re-admission rates.

\section{Diagnostic and prognostic of renal dysfunction}

\subsection{NGAL}

Acute renal failure is a prevalent complication of many disease states and is associated with significant morbidity and mortality. It was not until 2004 that a consensus regarding classification of stages of renal injury was developed. Bellomo et al. ${ }^{[29]}$ created the Risk-Injury-Failure-Loss-End stage kidney disease (RIFLES) criteria based on GFR, serum creatinine, and urine output. The risk, injury, and failure categories are associated with a high sensitivity while the loss and end stage kidney disease categories are highly specific. A patient may fall into one of the categories with a change in creatinine, urine output, or both. A patient is considered to be in chronic kidney disease (CKD) with a GFR $<60 \mathrm{~mL} / \mathrm{min}$ per $1.73 \mathrm{~m}^{2}$ for three months or more. Alternatively, any ongoing (at least three months) structural or functional abnormality of the kidney, regardless of GFR but that can be detected by pathological abnormalities or specific markers is considered CKD. ${ }^{[22]}$ These definitions have greatly aided physicians identifying disease states at a point where prognostically meaningful management can be instituted.

The kidney and heart are greatly affected by pathology of each other. Acute or chronic disease states of one organ may induce acute or chronic damage of the other. The term cardiorenal syndrome (CRS) describes this reciprocal process. There are five types of CRS, each discerned by the inciting disease process and the consequent dysfunction that ensues. ${ }^{[30]}$ Currently, as indicated by the RIFLEs criteria, creatinine is one of the major markers used to detect kidney dysfunction. However, several aspects of creatinine make it less than optimal. Many factors affect creatinine independent of kidney function including: age, gender, muscle mass and metabolism, medications, and level of hydration. ${ }^{[29,30]}$ Serum creatinine often does not rise until steady state equilibrium is reached, which in cases of injury could be days after the initial insult occurred. ${ }^{[29-32]}$ This decreases its sensitivity for acute changes in function. Additionally, many preventative and restorative measures for kidney injury are best if instituted immediately after the inciting event occurs, which is well before creatinine rises. ${ }^{[33-35]}$ In order to better assess kidney function, a marker that is more sensitive and convenient to measure at the bedside is necessary. Over the years, many markers have been suggested of which NGAL seems to have garnered much attention.

\subsubsection{NGAL in AKI}

NGAL was first purified and identified in 1993 by Kjeldsen et al. ${ }^{[36]}$ This small $25-\mathrm{kDa}$ protein with 178 amino acids belongs to the lipocalin family of proteins. The lipocalin family is a group of proteins that are otherwise diverse in sequence but share a specific conserved structural beta-barrel motif consisting of eight antiparallel strands joined by hydrogen bonds throughout. This core sequence serves to encircle the central binding pore specific for a variety of hydrophobic molecules, cell-surface receptors, and soluble macromolecules. ${ }^{[36]}$ NGAL has been shown to be involved in immune modulation, inflammation, and neoplastic transformation through macromolecule complexing and expression in immature neutrophils and epithelial cells. ${ }^{[37,38]}$ Broadly speaking, NGAL is physiologically expressed and secreted in the lung, kidney, trachea, stomach, and colon. Grenier et al. ${ }^{[39]}$ evaluated the performance of the ARCHITECT urine NGAL assay. They determined that the ARCHITECT assay was a reliably and reproducible assay to determine urine NGAL.

NGAL is thought to be bacteriostatic in pathological states by forming complexes with iron-binding siderophores and thus starving metabolically active bacteria. Additionally, serum and urine NGAL have been shown to increase dramatically in renal failure heralding its potential role as a marker for kidney injury. ${ }^{[40-43]}$ In a large study involving human and animal models, serum and urine NGAL were 7.3 and 25 -fold increased respectively in acute tubular necrosis compared to normal. Immunofluorescence was NGAL positive in $50 \%$ of the cortical tubules of kidneys with acute tubular necrosis compared to control kidney sections. ${ }^{[44]}$ Post-transplantation, NGAL is indicative of acute kidney injury. It correlates well with post-operative peak creatinine and necessitation of dialysis suggesting its potential involvement in routine cadaveric kidney allografts to predict kidney injury. ${ }^{[4,46]}$ ROC analysis with a urine NGAL cutoff of 50 $\mu \mathrm{g} / \mathrm{L}$ in predicting acute kidney injury in children post cardiopulmonary bypass produced AUC of $0.998 .{ }^{[38]}$ In 
adult patients, significantly higher urinary and serum NGAL were seen just after cardiac surgery, peaking at $3 \mathrm{~h}$, in patients who later developed acute renal dysfunction. In these patients, creatinine only peaked on post-operative day four. NGAL values dramatically decreased post-surgery in patients who did not develop acute renal dysfunction.

Ling et al. ${ }^{[47]}$ introduced the potential of urinary NGAL as a marker for contrast-induced nephropathy (CIN) $24 \mathrm{~h}$ after the procedure. Hirsh et al. ${ }^{[48]}$ found that a cutoff of $100 \mathrm{ng} / \mathrm{mL}$ predicted CIN in children with a sensitivity of 0.73 and specificity of 1 (urine NGAL AUC: 0.92, plasma NGAL AUC: 0.91 , both $P<0.0001$ ).

\subsubsection{NGAL in HF}

Recently, investigators have broken the confines of NGAL as purely a renal marker and explored the role of NGAL in cardiovascular pathology. ${ }^{[49]}$ Hypertension is a major risk factor of cardiovascular disease and renal injury is a known sequela of chronic elevated arterial pressure. A recent study by Malyszko et al. ${ }^{[50]}$ looked at the effect of hypertension on NGAL levels in patients with normal creatinine and stable coronary artery disease (CAD). NGAL values were higher in those with hypertension compared to controls (normotensives). In multivariate analysis, serum NGAL correlated with serum creatinine, urine NGAL, duration of hypertension, GFR, cystatin $\mathrm{C}$, and other parameters. Interestingly, serum creatinine was relatively similar between hypertensives and normotensives; however GFR was significantly decreased in hypertensives, indicating presence of renal injury where originally none was detected. This discrepancy highlights the potential benefit of tracking NGAL levels in patients with multiple risk factors for kidney damage, namely hypertension and CAD.

Amongst the geriatric population, CHF continues to be a pressing concern. Many patients acutely decompensate several times throughout disease progression and contribute to the greater than one million hospital admission annually. ${ }^{[51]}$ In addition, worsening renal function is a very real complication of $\mathrm{CHF}$ and is known to be an independent predictor of morbiddity and mortality as well as higher medical costs and longer hospital stays. ${ }^{[52]}$ It is in evaluating renal function of patients who acutely decompensate where NGAL has the greatest potential for creating a paradigm shift in management and prognostication.

Aghel et al. ${ }^{[53]}$ found that among patients admitted for acute decompensated HF, those who subsequently developed worsening renal function (defined as creatinine rise $>0.3 \mathrm{mg} / \mathrm{dL}$ ) had significantly higher NGAL values than those who maintained steady renal function (194 ng/mL vs. $128 \mathrm{ng} / \mathrm{mL}, P=0.001)$. Patients with an admission NGAL $>140 \mathrm{ng} / \mathrm{mL}$ were at 7.4fold increased risk of developing worsening kidney function during hospitalization (sensitivity $=86 \%$, specificity $=54 \%$ ), validating the accuracy with which NGAL can predict kidney injury.

Many studies have also looked at how NGAL compares with creatinine in detecting AKI. A meta-analysis by Haase et al. ${ }^{[54]}$ demonstrated that NGAL was an early predictor of subclinical AKI with early elevations in NGAL levels compared to serum creatinine. Moreover, in-patient mortality was highest in those patients with elevated NGAL levels with or without elevated serum creatinine.

Another study confirmed that increased NGAL along with NT-proBNP significantly correlated well with increased serum creatinine $(r=0.26, P=0.006)$ and decreased GFR $(r=-0.29, P=0.002)$ in patients with CHF compared to matched controls. ${ }^{[40]}$ Many studies have shown that NGAL rises 24 to $48 \mathrm{~h}$ before creatinine and thus shows promise in being a powerful marker for early detection of kidney damage. ${ }^{[55]}$ Dent et al. ${ }^{[55]}$ demonstrated that in patients undergoing cardiopulmonary bypass, NGAL detected AKI within $2 \mathrm{~h}$ where as rise in serum creatinine levels were delayed to 2-3 days. NGAL was the strongest indicator of AKI in the acute phase with a ROC-AUC of 0.96 in detecting AKI. Moreover, using a cut-off of $150 \mathrm{ng} / \mathrm{mL}$ to detect AKI, NGAL achieved $84 \%$ sensitivity and $94 \%$ specificity.

NGAL is a robust marker for detecting early AKI and carries high diagnostic and prognostic utility not only in patients with acute HF presenting to the emergency department with AKI but also in hospitalized patients with HF.

\subsection{Cystatin C}

Another novel marker rapidly emerging in the early detection of AKI with performance superior to creatinine is Cystatin C. The diagnostic utility of Cystatin $\mathrm{C}$ is well documented in the acute setting wherein early detection of worsening renal function is key to prevent progression to cardiorenal syndrome. ${ }^{[56-58]}$ Cystatin $\mathrm{C}$ is an endogenous cysteine proteinase inhibitor. Superiority of Cystaitn $\mathrm{C}$ over other markers of worsening renal function [marked by (estimated glomerular filtration rate) eGFR $<60 \mathrm{~mL} / \mathrm{min}$ ] lie with its ability to remain unbound to protein and being able to be freely filtered across the glomeruli. ${ }^{[57]}$

Most of the studies testing performance of urinary versus serum Cystatin markers have demonstrated a superior utility of the latter. Soto et al. ${ }^{[59]}$ conducted a study to evaluate diagnostic potential of Cystatin $\mathrm{C}$ for detecting $\mathrm{AKI}$ in patients presenting to the emergency department. Of the 616 enrolled, $21.1 \%$ had AKI (defined as serum creatinine $\geq 0.3 \mathrm{mg}$ increase from baseline as per RIFLE and AKIN criteria) with investigators carrying out serial measures at 5 time-points $(0$, 6, 12, 24, 48 h). In detecting AKI, Cystatin $\mathrm{C}$ achieved an AUC of $>0.86$ comparable to serum creatinine. Moreover, 
serum Cystatin $\mathrm{C}$ was able to discriminate between those with initial elevations in serum creatinine which returned to baseline in $48 \mathrm{~h}$ and those that went on to develop AKI.

Haase et al., ${ }^{[60]}$ demonstrated that plasma NGAL and serum Cystatin $\mathrm{C}$ were the strongest predictors of developing AKI (defined as per RIFLE criteria) in patients post-cardiac surgery. Cystatin $\mathrm{C}$ achieved an AUC of 0.76 , compared to NGAL 0.77 in detecting AKI on arrival. Overall, Cystatin C and NGAL performed better in detecting early AKI and predicting length of stay.

Several studies thus far have successfully evaluated the performance of Cystatin $\mathrm{C}$ in detecting early $\mathrm{AKI}$, a clinical entity that needs to be dealt with rapidly to prevent progression to cardiorenal syndrome in patients with acute HF.

\section{Prognostic markers}

\subsection{Procalcitonin}

Procalcitonin has been established as a biomarker indicative of ongoing inflammation and infection. The use of procalcitonin has been suggested to aid in diagnosis and prognosis of patients with microbial infections. Several studies thus far have defined procalcitonin as an acute phase reactant, superior to conventional inflammatory markers such as C-reactive protein (CRP) and cytokines. ${ }^{[61-65]}$ Human procalcitonin has a molecular weight of $14.5 \mathrm{kDa}$, a 116 amino acid structure and is encoded by the CALC-I gene located on the short arm of chromosome $11 .{ }^{\text {[66-69] }}$ In healthy individuals, post-translational processing of procalcitonin in thyroidal C-cells produces biologically active mature calcitonin. ${ }^{[61,64,69]}$ In infective conditions, procalcitonin mRNA up-regulation is seen in neuroendorine cells, parenchymal cells (adipose tissue) and peripheral blood mononuclear cells (PBMC) in response to endotoxin release or mediated via pro-inflammatory cytokines such as tumor necrosis factor (TNF), IL-6 and IL-8. ${ }^{[65,68-70]}$

\subsubsection{Procalcitonin in $\mathrm{HF}$}

Picariello et al. ${ }^{[71]}$ assessed 52 patients with acute coronary syndrome to establish if procalcitonin levels correlated with CRP as a marker for inflammation. Patients were divided into 3 sub-groups, cardiogenic shock (group A), uncomplicated STEMI (group B) and NSTEMI/UA (group C). Results revealed that procalcitonin levels were significantly higher $(>0.5 \mathrm{ng} / \mathrm{mL})$ specifically in group A compared to the other groups $(6.61 \mathrm{ng} / \mathrm{mL}, P<0.001)$, whereas CRP levels were significantly elevated in all three groups. Logistic regression showed that procalcitonin and CRP levels had no significant correlation in group A patients, suggesting that procalcitonin levels were more sensitive to and indicative of severe inflammatory process as seen in patients with cardiogenic shock.
The role of procalcitonin in predicting cardiovascular mortality in 2,131 patients with $\mathrm{CAD}$ showed that procalcitonin levels were significantly elevated in those with cardiovascular events on follow up $(0.016 \mathrm{ng} / \mathrm{mL}, P=0.0085)$ and in those that died from cardiovascular causes $(0.021 \mathrm{ng} / \mathrm{mL}, P<$ 0.0001). ${ }^{[72]}$ Kaplan-Meir analysis revealed a high event rate and cardiovascular mortality in those with procalcitonin levels $>0.023 \mathrm{ng} / \mathrm{mL}$. Elevated procalcitonin levels were found to be significantly associated with body mass index $(\mathrm{BMI})>30 \mathrm{~kg} / \mathrm{m}^{2}(P<0.0001)$, male gender $(P<0.0001)$ and diabetes mellitus $(P<0.0001)$. Furthermore, procalcitonin levels independently predicted future cardiovascular mortality on Cox regression analysis (HR: 1.34 ; $95 \% \mathrm{CI}$ : $1.08-1.65, P=$ 0.0070). According to the study, although the prognostic capabilities of procalcitonin surpassed conventional risk markers, it did not provide any additional information when compared with CRP. Procalcitonin levels were elevated in those with severe myocardial damage and associated with 6-month mortality suggesting that procalcitonin dynamics, especially in non-infective conditions and in patients with cardiovascular diseases, were influenced by severe myocyte injury resulting from ischemia. ${ }^{[72]}$

Often patients that present to the emergency department with congestive signs of HF have superimposed pneumonia which is hard to distinguish based on laboratory tests. A follow-up study using the BACH cohort analyzed the diagnostic and prognostic performance of procalcitonin in patients with pneumonia presenting to the emergency department with shortness of breath. ${ }^{[73]}$ The results revealed that procalcitonin added to the physicians clinical decision making in the emergency department, increasing their accuracy to $>83 \%$ for diagnosing pneumonia. Also, patients with high procalcitonin levels and untreated with antibiotics had the worst prognosis. Procalcitonin levels were obtained in $99.4 \%$ of patients and had an inter-quartile range from $0.05 \mathrm{ng} / \mathrm{mL}$ to $0.13 \mathrm{ng} / \mathrm{mL}$, a median of $0.07 \mathrm{ng} / \mathrm{mL}$ and $<0.05 \mathrm{ng} / \mathrm{mL}$ in healthy subjects. Procalcitonin also added to the diagnostic value of chest X-ray, increasing the AUC from 0.798 to 0.864 for diagnosing pneumonia.

\subsubsection{Procalcitonin in impaired renal function}

Patients with $\mathrm{CHF}$ often have impaired renal function necessitating dialysis, occurring as result of acute/chronic renal failure or secondary to cardiac or systemic infection. Managing such patients is always a daunting task. HergetRosenthal et al. ${ }^{[74]}$ evaluated 68 patients with end-stage renal failure or acute renal failure requiring hemodialysis prospectively to assess the diagnostic performance of procalcitonin to other conventional markers in detecting infection/sepsis. Eighty nine percent patients were correctly classified based on procalcitonin levels $>1.5 \mathrm{ng} / \mathrm{mL}$ as having severe infection or sepsis. A cut-off of $1.5 \mathrm{ng} / \mathrm{mL}$ achieved highest sensitivity 
$(89 \%)$, highest specificity (81\%) and accuracy $(85 \%)$ compared to CRP and total white cell count in diagnosing severe infection or sepsis. In the absence of infection, rising procalcitonin levels are due to reduced renal elimination. A study conducted on patients with chronic kidney disease without signs of infection or inflammation receiving renal replacement therapy showed that procalcitonin levels were conversely proportional to renal function and increased in parallel to depleting renal function. ${ }^{[75]}$ The study also assessed the role of PBMC in secreting procalcitonin in patients with reduced renal function and showed that PBMC's secreted higher amounts of procalcitonin in such patients.

Results from the BACH trial follow-up study showed that procalcitonin levels taken during follow-up identified patients requiring antibiotic use (procalcitonin levels $>0.21 \mathrm{ng} / \mathrm{mL}$, $P<0.01$ ), enabling adequate treatment of these patients which could have been potentially missed. ${ }^{[73]}$ Patients with procalcitonin levels $>0.21 \mathrm{ng} / \mathrm{mL}$ had a lower survival rate (80.5\% compared to $92 \%$ in those with procalcitonin $<0.05$ $\mathrm{ng} / \mathrm{mL}$ ). As mentioned earlier, distinguishing patients with acute HF and superimposed pneumonia can be challenging, this study showed that procalcitonin levels $(>0.1 \mathrm{ng} / \mathrm{mL})$ in addition to MR-proANP ( $>350 \mathrm{pmol} / \mathrm{L}$ ) were able to accurately diagnose those with community acquired pneumonia and concomitant acute HF. Thus, combining procalcitonin with MR-proANP proved useful in differentiating acute HF patients with pneumonia.

\subsection{Mid-Region pro-ADM (MR-proADM)}

ADM, a 52-amino-acid peptide, was first discovered in 1993 when it was isolated from a pheochromocytoma. ADM is homologous to the copeptin family and is expressed by many endothelial tissues throughout the body including the adrenal medulla, lungs, kidneys, gastrointestinal organs, and heart. ${ }^{[73-78]}$ $\mathrm{ADM}$ is secreted as an inactive precursor (pro-ADM) and subsequently cleaved into the active form where it acts as a potent vasodilator through the nitric oxide pathway and increases diuresis and natriuresis. ${ }^{[77-79]} \mathrm{ADM}$ has been shown to be a promising biomarker. Recent studies have shown that ADM levels increase after cardiac events, and its plasma levels have been shown to be inversely related to left ventricular function. However, the use of ADM as a biomarker has been hindered over the years due to its short plasma half-life of 22 min, making an accurate measurement difficult to attain. Recently, a more stable product of the pro-ADM peptide has been discovered and shown to be a promising prognostic tool, MR pro-ADM, a surrogate peptide of ADM. ${ }^{[80]}$

Recent studies have shown that MR pro-ADM is a promising prognostic tool for a wide variety of diseases, and in some cases, is a stronger prognostic indicator than the natriuretic peptides. In patients with symptoms of HF or a previous acute myocardial infarction, MR pro-ADM has been shown to be a good predictor of future cardiovascular events and death. ${ }^{[80]}$ This is further confirmed by the BACH study where it was shown that MR-proADM levels (cutoff of $1.985 \mathrm{nmol} / \mathrm{L}$ ) had a predictive accuracy for survival at 90 days of $73 \%$ in patients presenting to the emergency department with shortness of breath. This is in comparison to BNP which only had a predictive accuracy of $62 \% .{ }^{[81]}$

Another study by Haehling et al. ${ }^{[82]}$ showed that high levels of MR-proADM correlated with an increased risk of death within 12 months, independent of age, creatinine, left ventricular ejection fraction, NT-proBNP, and NYHA class. MR-proADM has also been shown to be useful in the prognosis of patients with non-ST elevation myocardial infarction. In an additional study, levels of MR-proADM were taken $36 \mathrm{~h}$ after the onset of symptoms and at discharge. It was determined that a high MR-proADM level at admission was associated with death and early mortality (better than GRACE score and NT-proBNP), while high levels at discharge were associated with HF readmission. ${ }^{[83]}$ Levels and predictive strength of MR-proADM have also been shown to differ based on the type and severity of HF. MR-proADM has been shown to be better as an independent predictor of death for patients with mild to moderate symptomatic HF and non-ischemic heart injury, thus showing that MR-proADM is a promising, yet situational biomarker. ${ }^{[84]}$ MR-proADM shows great promise as a potential cardiac biomarker, although certain characteristics should be taken into consideration.

While MR-proADM has been shown as a strong predictive marker for a variety of cardiac disease, it is also a biomarker for other diseases that present with shortness of breath, including chronic obstructive pulmonary disorder pneumonia, and pulmonary embolism. ${ }^{[85,86]}$ And while MR-proADM adds prognostic strength to NT-proBNP, it by no means can replace BNP and troponin as a diagnostic and prognostic tool. ${ }^{[81]}$ However, since MR-proADM levels have been shown to differ based on NYHA class and severity of HF, it has potential to help identify those patients that may benefit from more invasive therapy. Overall, MR-proADM shows great promise as an independent prognostic tool for cardiac disease, although further investigation should be performed to further clarify its use as a biomarker.

\subsection{Copeptin}

Arginine vasopressin (AVP), which is also known as antidiuretic hormone, is produced in the neurohypophysis of the paraventricular and supraoptic nuclei of the hypothalamus. An excess of AVP levels can affect cardiac contractility and vascular tone by inducing hyponatremia and edema in CHF. 
Its role in the progression of CHF and exacerbation of left ventricular dysfunction make it a viable target for the treatment of CHF. ${ }^{[87]}$ Due to its small size, fast clearance rate, and high fraction of the platelet-bound form, AVP is very difficult to measure in vitro. ${ }^{[74]}$ Additionally, it is unstable (half-life of 5-15 min) when extracted in plasma form, even at low storage temperatures $\left(-20^{\circ} \mathrm{C}\right) .{ }^{[88,89]}$ Thus, AVP levels can never be measured accurately and efficiently in a practical manner in a clinical setting. This is where a new biomarker, copeptin, comes into play.

AVP comes from a 166-amino acid-long precursor protein, preprovasopressin, which is composed of a signal peptide, AVP, neurophysin II, and copeptin. ${ }^{[88,90]}$ Copeptin (CT-proAVP), a 39-amino acid-long glycosylated peptide with a leucine-rich core segment, is named for the C-terminal portion of this precursor molecule. ${ }^{[88]}$ Given that the hormones are secreted stoichiometrically, the release of copeptin essentially mirrors that of AVP. ${ }^{[91]}$ However, copeptin has been shown to be a more stable counterpart, able to stand at room temperature in serum or plasma form without degrading significantly. ${ }^{[92,93]}$

\subsubsection{Copeptin in HF}

In an extension of the BACH study, ${ }^{[94]}$ copeptin was measured in 557 patients with acute HF. 90-day mortality was found to be greater in patients with increased copeptin levels $(P<$ 0.001, HR 3.85). The combined endpoints of mortality, readmissions, and emergency department visits were even more likely with these risen levels. Copeptin added to the prognostic value of other clinical predictors in patients with acute HF for 90-day increased mortality.

Other studies have also shown that copeptin alone can be superior to other biomarkers involved in HF. In the OPTIMAAL study, ${ }^{[95]}$ higher copeptin levels were found to have a significant association with mortality. ROC curves demonstrated that copeptin was a stronger predictor of mortality compared with both BNP and NT-proBNP. Changes of copeptin levels after one month significantly added prognostic information to the baseline value. In a multi-variable Cox proportional hazards model, a doubling of copeptin was related to a 1.83 times increased risk of mortality. Likewise, in a study conducted on 287 patients with acute dyspnea in the emergency department, copeptin was the strongest predictor for short-term mortality in all patients (HR 3.88, $P<0.001$ ), and especially in those with acute decompensated HF (HR 5.99, $P<0.0001$ ).

While copeptin can predict outcomes in HF patients, it has been proven that AVP can have physical effects on the heart as well. In the Kelly et al. ${ }^{[96]}$ study, copeptin was associated with left ventricular (LV) dysfunction, volumes, remodeling, and clinical HF post-acute myocardial infarction. Its levels were directly proportional with the wall motion index score, and inversely proportional to LV ejection faction at discharge and follow-up and with ventricular volumes at follow up. Patients with increasing LV end-systolic volume had greater levels of copeptin. There was also an increased risk in subjects with copeptin levels $>6.31 \mathrm{pmol} / \mathrm{L}$.

\subsubsection{Copeptin in renal dysfunction}

As described in the Bhandari et al. ${ }^{[91]}$ study, copeptin showed a significant correlation to eGFR in males $(r=-0.186$, $P<0.001)$ and a less defined correlation in females $(r=-0.097$, $P=0.095)$. In addition, copeptin was found to be involved in the development of urinary albumin excretion in patients. In the PREVEND study, ${ }^{[98]}$ urinary albumin excretion is twice as high in individuals within the upper quintiles of plasma copeptin than those in the lowest quintile. This high urinary albumin excretion in individuals with high copeptin levels is a reflection of the increase in permeability of the glomerular filter to albumin or a decrease in the reabsorption of albumin.

How AVP induces albuminuria is unclear, but there is an obvious connection between the two phenomena. An increase in AVP leads to an increase in urinary albumin excretion, a sign of early renal damage and progression of renal failure. ${ }^{[97]}$ This suggests an indirect involvement of AVP in kidney dysfunction.

Due to evidence of its participation in HF and renal failure from the previous studies, copeptin is a potential prognostic marker in cardiorenal syndrome. Its successor, AVP has been shown to contribute to the progression of cardiac and renal failure. In HF, AVP limits the contractility of the heart by inducing hyponatremia and edema. In renal dysfunction, AVP secretion gave rise to urinary albumin excretion and decreased glomerular filtration rate.

The CHART 2 study, ${ }^{[98]}$ investigated albuminuria, the link between cardiac and renal failure, in HF patients with preserved ejection fractions. This was a large study that enrolled 2,465 patients with overt HF with ejection fractions $\geq 50 \%$. A urinary dipstick test (UDT) was performed in each of these patients for trace of proteinuria. It was found that patients with eGFR $<60$ had more severe positive dipsticks compared with those with eGFR $\geq 60$. HF patients with preserved ejection fractions patients with positive UDT had significantly higher mortality compared with those with a negative UDT in each stratum of eGFR levels.

Copeptin is stoichiometrically released with AVP, which reflects urinary albumin excretion in a general population of patients. Copeptin may be used as a marker of albuminuria, which in turn can be used as a marker for cardiorenal syndrome.

\section{Conclusions}

The incoming of ultrasensitive assays allowing a faster interpretation along with a multi-marker strategy remain the 
Table 1. Strength of evidence for individual markers in diagnosis, risk stratification and guided treatment for acute heart failure.

\begin{tabular}{lccc}
\hline & Diagnostic capability & Risk Stratification & Biomarker guided treatment \\
\hline Natriuretic peptides & $+++^{\mathrm{a}}$ & ++ & ++ \\
Procalcitonin & $+++^{\mathrm{b}}$ & ++ \\
NGAL & $++^{\mathrm{c}}$ & ++ \\
Cystatin C & $+++^{\mathrm{c}}$ & ++ \\
MR-proADM & - & ++ \\
Copeptin & - & ++ \\
Troponin & +++ & +++ \\
\hline
\end{tabular}

NGAL: Neutrophil gelatinase-associated lipocalin; MR-proADM: Mid-regional pro Adrenomedullin; ${ }^{\mathrm{a}}$ Heart Failure; ${ }^{\mathrm{b}}$ Pulmonary Infection; ${ }^{\mathrm{c}}$ Acute Kidney Injury.

mainstay for managing HF patients. As any clinical test, care must be taken in the interpretation of biomarker levels subject to confounders. ${ }^{[99]}$ For instance, the presence of greyzone values can be complexing while interpreting $\mathrm{BNP} /$ NT-proBNP levels, as in such patients, their elevated levels are confounded by the presence of right sided HF, pulmonary embolism and diastolic failure. ${ }^{[100]}$ Studies have established that biomarkers by no means are stand-alone tests but simply additive tools to be used in diagnosis and risk stratification. Table 1 demonstrates the strength of evidence for all biomarkers in comparison to natriuretic peptides. Majority of HF patients initially present via the emergency department, and therein the role of biomarker testing has shown to improve clinical decision making and reduce future re-hospitalizations. Another facet of biomarker testing involves the serial monitoring of their levels to chart disease progression, thus, achieving better management of HF patients with poor renal function by reducing unwanted/over-use of pharmacological drugs. Preserving renal function is challenging in HF patients and the rationale behind the use of biomarkers in such instances lie in their ability to rapidly reflect ongoing pathophysiological changes. Inpatient complications need extensive treatment and markers such as procalcitonin has shown substantial promise by predicting clinical outcomes. The role of biomarkers has certainly evolved in the last decade and continues to evolve with growing research pointing towards their use in standard therapy.

\section{Disclosures}

Alan S. Maisel is a consultant for Alere and serves on the advisory board for Critical Diagnostics. He receives research support from Critical Diagnostics, Alere, Abbott, Nanosphere, and Brahms-Thermo Fisher Scientific. The remaining authors have not reported disclosures.

\section{References}

1 Ronco C, Cruz DN, Ronco F. Cardiorenal syndrome. Curr Opin Crit Care 2009; 15: 384-391.
2 Ronco $\mathrm{C}$, McCullough $\mathrm{P}$, Anker SD, et al. Cardio-renal syndromes: report from the consensus conference of the Acute Dialysis Quality Initiative. Eur Heart J 2010; 31: 703-711.

3 Hawkins R. New biomarkers of acute kidney injury and the cardio-renal syndrome. Korean J Lab Med 2011; 31: 72-80.

4 Vishwanathan G, Gilbert S. The cardiorenal syndrome: Making the Connection. Int J Nephrol 2011; 283137.

5 Carubelli V, Metra M, Lombardi C, et al. Renal dysfunction in acute heart failure: epidemiology, mechanisms and assessment. Heart Fail Rev 2012; 17: 271-282.

6 Cleland JCF, Carubelli V, Castiello T, et al. Renal dysfunction in acute and chronic heart failure: prevalence, incidence and prognosis. Heart Fail Rev 2012; 17: 133-149.

7 Smith GL, Lichtman JH, Bracken MB, et al. Renal impairment and outcomes in heart failure. J Am Coll Cardiol 2006; 47: 1987-1996.

8 De Luca L, Fonarrow GC, Adams Jr AF, et al. Acute heart failure syndromes: clinical scenarios and pathophysiologic targets. Heart Fail Rev 2007; 12: 97-104.

9 Weintraub NL, Collins SP, Pang PS, et al. Acute heart failure syndromes: Emergency Department Presentation, Treatment, and Disposition: Current Approaches and Future Aims. Circulation 2010; 122: 1975-1996.

10 Karasek J, Widimsky P, Ostadal P, et al. Acute heart failure registry from high-volume university hospital ED: comparing European and US data. Am J Emerg Med 2012; 30: 695-705.

11 Ronco C, McCullough P, Anker SD, et al. Cardio-renal syndromes: report from the consensus conference of the Acute Dialysis Quality Initiative. Eur Heart J 2010; 31: 703-711.

12 Cotter G, Felker M, Adams KF, et al. The pathophysiology of acute heart failure-Is it all about fluid accumulation? Am Heart J 2008; 155: 9-18.

13 Krumholz HM, Wang Y, Parent EM, et al. Quality of care for elderly patients hospitalized with heart failure. Arch Intern Med 1997; 27: 2242-2247.

14 Cowie MR, Jourdain P, Maisel A, et al. Clinical applications of B-type natriuretic peptide (BNP) testing. Eur Heart J 2003; 24: 17101-718.

15 Fonarow GC, Peacock WF, Phillips CO, et al. Admission B-type Natriuretic peptide levels and in-hopsital mortality in 
acute decompensated heart failure. J Am Coll Cardiol 2007; 49: 1943-1950.

16 Maisel A, Mueller C, Kirkwood A, et al. State of the art: Using natriuretic peptide levels in clinical practice. Eur $J$ Heart Fail 2008; 10: 824-839.

17 Maisel A, Hollander JE, Guss D, et al. Primary results of the rapid emergency department heart failure outpatient trial (REDHOT). J Am Coll Cardiol 2004; 44: 1328-1333.

18 Di Somma S, Magrini L, Pittoni V, et al. In-hospital percentage BNP reduction is highly predictive for adverse events in patients admitted for acute heart failure: the Italian RED Study. Crit Care 2010; 14: R116.

19 Vanderheyden M, Bartunek J, Goethals M. Brain and other natriuretic peptides: molecular aspects. Eur J Heart Fail 2004; 6: 261-268.

20 Van Kimmenade RR, Januzzi Jr JL, Baggish AL, et al. Amino-Terminal Pro-Brain Natriuretic Peptide, Renal Function, and Outcomes in Acute Heart Failure. J Am Coll Cardiol 2006; 48: 1621-1627.

21 Van Kimmenade RR, Januzzi Jr JL, Ellinor PT, et al. Utility of Amino-Terminal Pro-Brain Natriuretic Peptide, Galectin-3, and Apelin for the Evaluation of Patients with Acute Heart Failure. J Am Coll Cardiol 2006; 48: 1217-1224.

22 Januzzi JL, van Kimmenade R, Lainchbury J, et al. NT-proBNP testing for diagnosis and short-term prognosis in acute destabilized heart failure: an international pooled analysis of 1256 patients. Eur J Heart Fail 2006; 27: 330-337.

23 Morrow DA, Cannon CP, Jesse RL, et al. National Academy of Clinical Biochemistry Laboratory Medicine Practice Guidelines: clinical characteristics and utilization of biochemical markers in acute coronary syndromes. Clin Chem 2007; 53: 552-574.

24 Sezer S, Karakan S, Ozdemir N. Increased cardiac troponin T levels are related to inflammatory markers and various indices of renal function in chronic renal disease patients. Ren Fail 2012; 34: 454-459.

25 Daubert MA, Jeremias A. The utility of troponin measurement to detect myocardial infarction: review of the current findings. Vasc Health Risk Manag 2010; 6: 691-699.

26 Kitagawa M, Sugiyama H, Morinaga H, et al. Serum highsensitvity cardiac troponin $\mathrm{T}$ is a significant biomarker of leftventricular diastolic dysfucntion in subjects with non-diabetic chronic kidney disease. Nephron Extra 2011; 1: 166-177.

27 Nishio Y, Sato Y, Taniguchi R, et al. Cardiac troponin T vs. ther biochemical markers in patients with congestive heart failure. Circ J 2007; 71: 631-635.

28 De Filippi CR, De Lemos JA, Christenson RH, et al. Association of serial measures of cardiac troponin $\mathrm{T}$ using a sensitive assay with incident heart failure and cardiovascular mortality in older adults. JAMA 2010; 304: 2494-2502.

29 Bellomo R, Ronco C, Kellum J, et al. Acute renal failuredefinition, outcome measures, animal models, fluid therapy and information technology needs: The second international consensus conference of the acute dialysis quality initiative
(ADQI) group. Critical Care 2004; 8: R204-R212.

30 Parrinello G, Paterna S, Di Pasquale P, et al. The usefulness of bioelectrical impedance analysis in differentiating dyspnea due to decompensated heart failure. J Card Fail 2008; 14: 676-686.

31 Heymsfield SB, Wang ZM, Visser M, et al. Techniques used in the measurement of body composition: an overview with emphasis on bioelectrical impedance analysis. Am J Clin Nutr 1996; 64: 478S-484S.

32 Damman K, van Deursen VM, Navis G, et al. Increased central venous pressure is associated with impaired renal function and mortality in a broad spectrum of patients with cardiovascular disease. J Am Coll Cardiol 2009; 53: 582-588.

33 Levy AS, Eckardt KU, Tsukamoto Y, et al. Definition and classification of chronic kidney disease: A position statement from kidney disease: Improving global outcomes (KDIGO). Kidney Int 2005; 67: 2089-2100.

34 Ronco C, Haapio M, House AA, et al. Cardiorenal syndrome. $J$ Am Coll Cardiol 2008; 52: 1527-1539.

35 Devarajan P. Neutrophil gelatinase-associated lipocalin (NGAL): A new marker of kidney disease. Scand J Clin Lab Invest Suppl 2008; 241: 89-94.

36 Kjeldsen L, Johnsen AH, Sengeløv H, et al. Isolation and primary structure of NGAL, a novel protein associated with human neutrophil gelatinase. J Biol Chem 1993; 268: 1042510432.

37 Flower DR. The lipocalin protein family: Structure and function. Biochem J 1996; 318: 1-14.

38 Goetz DH, Willie ST, Armen RS, et al. Ligand preference inferred from the structure of neutrophil gelatinase associated lipocalin. Biochemistry 2000; 39: 1935-1941.

39 Grenier FC, Ali S, Syed H, et al. Evaluation of the ARCHITECT urine NGAL assay: Assay performance, specimen handling requirements and biological variability. Clin Biochem 2010; 43: 615-620.

40 Damman K, van Veldhuisen DJ, Navis G, et al. Urinary neutrophil gelatinase associated lipocalin (NGAL), a marker of tubular damage, is increased in patients with chronic heart failure. Eur J Heart Fail 2008; 10: 997-1000.

41 Schmidt-Ott KM, Mori K, Li JY, et al. Dual action of neutrophil Gelatinase-Associated lipocalin. J Am Soc Nephrol 2007; 18: 407-413.

42 Bolignano D, Donato V, Coppolino G, et al. Neutrophil GelatinaseAssociated lipocalin (NGAL) as a marker of kidney damage. Am J Kidney Dis 2008; 52: 595-605.

43 Wagener G, Jan M, Kim M, et al. Association between increases in urinary neutrophil gelatinase-associated lipocalin and acute renal dysfunction after adult cardiac surgery. Anesthesiology 2006; 105: 485-491.

44 Mori K, Lee HT, Rapoport D, et al. Endocytic delivery of lipocalin-siderophore-iron complex rescues the kidney from ischemia-reperfusion injury. $J$ Clin Invest 2005; 115: 610-621.

45 Mishra J, Ma Q, Kell C, et al. Kidney NGAL is a novel early 
marker of acute injury following transplantation. Pediatr Nephrol 2006; 21: 856-863.

46 Mishra J, Dent C, Tarabishi R, et al. Neutrophil gelatinaseassociated lipocalin (NGAL) as a biomarker for acute renal injury after cardiac surgery. The Lancet 2005; 365: 1231-1238.

47 Ling W, Zhaohui N, Ben H, et al. Urinary IL-18 and NGAL as early predictive biomarkers in contrast-induced nephropathy after coronary angiography. Nephron Clin Pract 2008; 108: 176-181.

48 Hirsch R, Dent C, Pfriem H, et al. NGAL is an early predictive biomarker of contrast-induced nephropathy in children. Pediatr Nephrol 2007; 22: 2089-2095.

49 Bolignano D, Coppolino G, Lacquaniti A, et al. From kidney to cardiovascular diseases: NGAL as a biomarker beyond the confines of nephrology. Eur J Clin Invest 2010; 40: 273-276.

50 Malyszko J, Bachorzewska-Gajewska H, Malyszko JS, et al. Serum neutrophil gelatinase-associated lipocalin as a marker of renal function in hypertensive and normotensive patients with coronary artery disease. Nephrology 2008; 13: 153-156.

51 Viswanathan G, Gilbert S. The cardiorenal syndrome: Making the connection. Int J Nephrol 2011; 283137.

52 Herout PM, Harshaw Q, Phatak H, et al. Impact of worsening renal function during hospital admission on resource utilization in patients with heart failure. Am J Cardiol 2010; 106: 1139-1145.

53 Aghel A, Shrestha K, Mullens W, et al. Serum neutrophil gelatinase-associated lipocalin (NGAL) in predicting worsening renal function in acute decompensated heart failure. $J$ Card Fail 2010; 16: 49-54.

54 Haase M, Devarajan P, Haase-Fielitz A, et al. The outcome of neutrophil gelatinase-associated lipocalin-positive subclinical acute kidney injury: a multicenter pooled analysis of prospective studies. J Am Coll Cardiol 2011; 57: 1752-1761.

55 Dent CL, Ma Q, Dastrala S, et al. Plasma neutrophil gelatinaseassociated lipocalin predicts acute kidney injury, morbidity and mortality after pediatric cardiac surgery: a prospective controlled cohort study. Crit Care 2007; 11: R127.

56 Haase-Fielitz A, Bellomo R, Devarajan P, et al. Novel and conventional serum biomarkers predicting acute kidney injury in adult cardiac surgery-A prospective cohort study. Crit Care Med 2009; 37: 553-560.

57 Zhang Z, Baolong L, Sheng X, et al. Cystatin C in prediction of acute kidney injury: A systematic review and meta-analysis. Am J Kidney Dis 2011; 58: 356-365.

58 Damman K, van der Harst P, Smilde TDJ, et al. Use of cystatin $\mathrm{C}$ levels in estimating renal function and prognosis in patients with chronic systolic heart failure. Heart 2012; 98: 319-324.

59 Soto K, Coelho S, Rodrigues B, et al. Cystatin C as a marker of acute kidney injury in the emergency department. Clin $J$ Am Soc Nephrol 2010; 5: 1745-1754.

60 Haase M, Bellomo R, Devarajan P, et al. Novel biomarkers early predict the severity of acute kidney injury after cardiac surgery in adults. Ann Thorac Surg 2009; 88: 124-130.
61 Assicot M, Gendrel D, Carsin H, et al. High serum procalcitonin concentrations in patients with sepsis and infection. The Lancet 1993; 341: 515-518.

62 Carrol ED, Thomson APJ, Hart CA. Procalcitonin as a marker of sepsis. Int J Antimicrob Agents 2002; 20: 1-9.

63 Nijsten M, Olinga P, Hauw TT, et al. Procalcitonin behaves as a fast responding acute phase protein in vivo and in vitro. Crit Care Med 2000; 28: 458-461.

64 Meisner M, Adina H, Scmidt J. Correlation of procalcitonin and C-reactive protein to inflammation, complications, and outcome during the intensive care unit course of multiple-trauma patients. Crit Care 2006; 10: R1.

65 Christ-Crain M, Muller B. Biomarkers in respiratory tract infections: diagnostic guides to antibiotic prescription, prognostic markers and mediators. Eur Respir J 2007; 30: 556-573.

66 Russworm S, Wiederhold M, Oberhoffer M, et al. Molecular aspects and natural source of procalcitonin. Clin Chem Lab Med 1999; 37: 789-797.

67 Ittner L, Born W, Rau B, et al. Circulating procalcitonin and cleavage products in septicemia compared with medullary thyroid carcinoma. Eur J Endocrinol 2002; 147: 727-731.

68 Meisner M. Pathobiochemsitry and clinical use of procalcitonin. Clin Chim Acta 2007; 323: 17-29.

69 Jin M, Khan AI. Procalcitonin: Uses in the clinical laboratory for the diagnosis of sepsis. Lab Med 2010; 41: 173-177.

70 Linscheid P, Seboek D, Schaer DJ, et al. Expression and secretion of procalcitonin and calcitonin gene-related peptide by adherent monocytes and by macrophage-activated adipocytes. Crit Care Med 2004; 32:1715-1721.

71 Picariello C, Lazzeri C, Chiostri M, et al. Procalcitonin in patients with acute coronary syndromes and cardiogenci shock submitted to percutaneous coronary intervention. Intern Emerg Med 2009; 4: 403-408.

72 Sinning CR, Sinning J-M, Schulz A, et al. Association of serum procalcitonin with cardiovascular prognosis in coronary artery disease. Circ J 2011; 75: 1184-1191.

73 Maisel A, Neath SX, Landsberg J, et al. Use of Procalcitonin (PCT) for the diagnosis of pneumonia in patients presenting with a chief complaint of dyspnoea: Results from the BACH (Biomarkers in Acute Heart Failure) trial. Eur J Heart Fail 2012; 14: 278-286.

74 Herget-Rosenthal S, Marggraf G, Pietruck F, et al. Procalcitonin for accurate detection of infection in hemodialysis. Nephrol Dial Transplant 2001; 16: 975-979.

75 Herget-Rosenthal S, Marggraf G, Hirsch T, et al. Modulation and source of procalcitonin in reduced renal function and renal replacement therapy. Scand J Immunol 2005; 61: 180-186.

76 Yamaguchi T, Baba K, Doi Y, et al. Effect of adrenomedullin on aldosterone secretion by dispersed rat adrenal zona glomerulosa cells. Life Sci 1995; 56: 379-387. 
77 Yandle T, Troughton R. Improving risk stratification in heart failure: a role for new biomarkers? Eur J Heart Fail 2010; 12: 315-318.

78 Rademaker MT, Cameron VA, Charles CJ, et al. Adrenomedullin and heart failure. Regul Pept 2003; 112: 51-60.

79 Chan D, Leong N. Biomarkers in acute myocardial infarction. BMC Med 2010; 8: 34.

80 Klip I, Voors A, Anker S, et al. Prognostic value of mid-regional pro-adrenomedullin in patients with heart failure after an acute myocardial infarction. Heart 2001; 97: 892-898.

81 Maisel A, Mueller C, Nowak R, et al. Midregion prohormone adrenomedullin and prognosis in patients presenting with acute dyspnea, results of the BACH trials. J Am Coll Cardiol 2011; 58: 1057-1067.

82 Haehling S, Filippatos G, Papassotiriou J, et al. Mid-regional pro-adrenomedullin as a novel predictor of mortality in patients with chronic heart failure. Eur J Heart Fail 2010; 12: 484- 491.

83 Katayama T, Nakashima H, Furodono S, et al. Evaluation of nuerohumoral activation (adrenomedullin, BNP, catecholamines, etc.) in patients with acute myocardial infarction. Intern Med 2004; 43: 1015-1022.

84 Dhillon O, Khan S, Narayan $\mathrm{H}$, et al. Prognostic value of mid-regional pro-adrenomedullin levels taken on admission and discharge in non-st-elevation myocardial infarction, The LAMP (Leicester Acute Myocardial Infarction Peptide) II Study. J Am Coll Cardiol 2010; 56: 125-133.

85 Schuetz P, Wolbers M, Christ-Crain M, et al. Prohormones for prediction of adverse medical outcome in community-acquired pneumonia and lower respiratory tract infections. Crit Care 2010; 14: R106.

86 Kruger S, Ewig S, Giersdorf S, et al. Cardiovascular and inflammatory biomarkers to predict short- and long-term survival in community-acquired pneumonia. Am J Respir Crit Care Med 2010; 182: 1426-1434.

87 Chatterjee K. Neurohormonal activation in congestive heart failure and the role of vasopressin. Am J Cardiol 2005; 95: 8B-13B.

88 Dabla PK, Dabla V, Arora S. Co-peptin: Role as a novel biomarker in clinical practice. Clin Chim Acta 2011; 412: 22-28.

89 Lin S, Yokoyama H, Rac VE, et al. Novel biomarkers in diagnosing cardiac ischemia in the emergency department: A systematic review. Resuscitation 2012; 83: 684-691.

90 Morgenthaler NG, Struck J, Jochberger S, et al. Copeptin: clinical use of a new biomarker. Trends Endocrinol Metab 2008; 19: 43-49.

91 Bhandari SS, Loke I, Davies JE, et al. Gender and renal function influence plasma levels of copeptin in healthy individuals. Clin Sci 2009; 116: 257-263.

92 Morgenthaler NG, Struck J, Alonso C, et al. Assay for the measurement of copeptin, a stable peptide derived from the precursor of vasopressin. Clin Chem 2006; 52: 112-119.

93 Szinnai G, Morgenthaler NG, Berneis K, et al. Changes in plasma copeptin, the c-terminal portion of arginine vasopressin during water deprivation and excess in healthy subjects. J Clin Endocrinol Metab 2007; 92: 3973-3978.

94 Maisel A, Xue Y, Shah K, et al. Increased 90-Day Mortality in Patients With Acute Heart Failure With Elevated Copeptin: Secondary Results From the Biomarkers in Acute Heart Failure (BACH) Study. Circ Heart Fail 2011; 4: 613-620.

95 Voors AA, von Haehling S, Anker SD, et al. OPTIMAAL Investigators. C-terminal provasopressin (copeptin) is a strong prognostic marker in patients with heart failure after an acute myocardial infarction: results from the OPTIMAAL study. Eur Heart J 2009; 30: 1187-1194.

96 Kelly D, Squire IB, Khan SQ, et al. C-terminal provasopressin (copeptin) is associated with left ventricular dysfunction, remodeling, and clinical heart failure in survivors of myocardial infarction. J Card Fail 2008; 14: 739-745.

97 Meijer E, Bakker SJ, Halbesma N, et al. Copeptin, a surrogate marker of vasopressin, is associated with microalbuminuria in a large population cohort. Kidney Int 2010; 77: 29-36.

98 Miura M, Shiba N, Nochioka K, et al. Urinary albumin excretion in heart failure with preserved ejection fraction: an interim analysis of the CHART 2 study. Eur $J$ Heart Fail 2012; 14: 367-376.

99 Katan M \& Christ-Crain M. The stress hormone copeptin: a new prognostic biomarker in acute illness. Swiss Med Wkly 2010; 140: 13101 .

100 Maisel AS. Natriuretic peptides: what's on the horizon. Future Cardiol 2008; 4: 107-109. 